\title{
Influence of an AGN complex photon field on the jet bulk Lorentz factor through Compton rocket effect
}

\author{
Thomas Vuillaume, Gilles Henri and Pierre-Olivier Petrucci \\ Université Grenoble Alpes, IPAG, F-38000 Grenoble, France \\ \& CNRS, IPAG, F-38000 Grenoble, France \\ email: thomas.vuillaume@obs.ujf-grenoble.fr
}

\begin{abstract}
In this work, we study the acceleration of hot plasma to relativistic speed through the Compton rocket effect which is viable in the two-flow paradigm.
\end{abstract}

\section{Introduction}

It is now widely admitted that AGN's jets hold relativistic flows. First evidences go back to the 70's with the observation of superluminal motions (Cohen et al. 1971) which are only possible for actual speeds of $0.7 c$ at least. However, a lot of questions on the speed of these flows remain. Mainly, we still do not know the mechanism driving them to relativistic speeds or neither do we know the spatial distribution of these speeds in the flows. They can be characterized by their bulk Lorentz factor $\Gamma_{b}=\left(1-\beta_{b}^{2}\right)^{-1 / 2}$ rather than their speed $V_{b}$ with $\beta_{b}=V_{b} / c$. In some studies, the distribution of Lorentz factors appears to follow power laws with an accelerating and/or a decelerating phase (Marscher 1980, Ghisellini et al. 1985, Boutelier et al. 2008).

Our work takes place in the two-flow paradigm (Sol et al. 1989) where the jet is composed of a mildly relativistic sheath, filled with $e^{-} / p^{+}$and an ultra-relativistic spine composed of $e^{-} / e^{+}$pairs responsible for most of the emission. The outer jet acts as an energy reservoir for the particles of the spine, which will be continuously thermalized along the jet via the second order Fermi process. This is in agreement with diffuse X-ray emission observed in FRI which favors a distributed particle acceleration rather than localized shocks (Hardcastle et al. 2007). In this paradigm, the plasma is subject to the Compton rocket effect which will naturally drive the flow to relativistic speeds.

\section{2. $\Gamma_{b} \&$ equilibrium}

Cheng \& O'Dell (1981) showed that "a plasma of relativistic particles exposed to an anisotropic radiation field acts as a rocket - a Compton rocket" because of the reaction force imposed by the inverse Compton radiation from the particles. In the Thomson regime, this force is proportional to the flux in the plasma rest frame $H^{*}=$ $\frac{1}{4 \pi} \int I_{\nu_{s}}^{*}\left(\Omega_{s}^{*}, \Gamma_{b}\right) \cos \theta_{s}^{*} \mathrm{~d} \Omega_{s}^{*} \mathrm{~d} \nu_{s}^{*}$ with the quantities in the rest frame: $I_{\nu_{s}}^{*}$ the specific intensity of the radiation reaching the plasma with a solid angle $\mathrm{d} \Omega^{*}$ and an angle $\theta_{s}^{*}$ defined in Figure 1 at a frequency $\nu_{s}^{*}$. Thus, the bulk of particles reaches an equilibrium velocity, which can be represented by the equilibrium bulk Lorentz factor $\Gamma_{e q}$, when $H^{*}=0$. To compute $\Gamma_{e q}$, we need to compute precisely the external photon field at any position of the plasma. Here, we model the AGN including three main sources of soft 


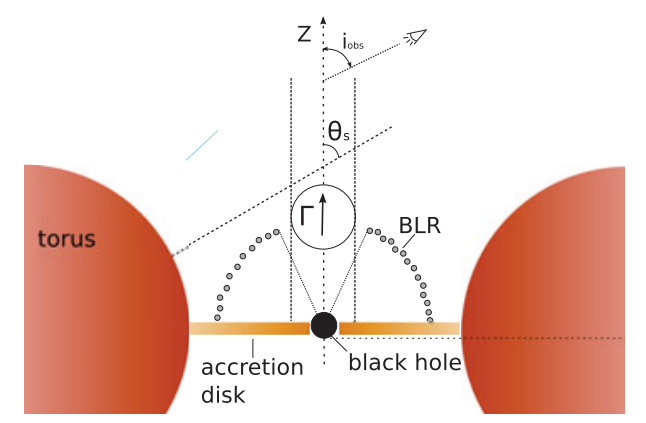

Figure 1. The big picture: sketch edge-on of the global model geometry (not to scale) with the accretion disk, the dusty torus and the BLR.

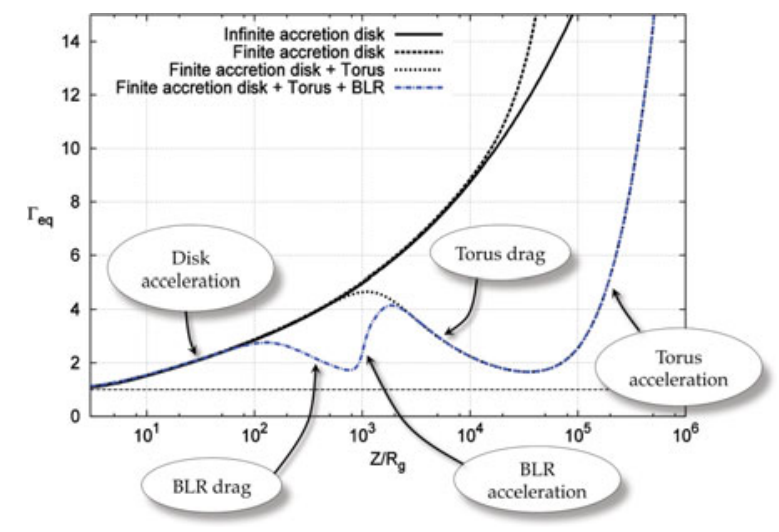

Figure 2. $\Gamma_{e q}$ resulting from the Compton rocket effect for different external photon sources. The geometry is described in Figure 1 with the following parameters: finite and infinite accretion disk have an inner radius $R_{i n}=3 R_{g}$. The finite disk has an outer radius $R_{\text {out }}=5 \times 10^{4} R_{g}$. $D_{\text {torus }}=10^{5} R_{g}, R_{\text {torus }}=5 \times 10^{4} R_{g}, R_{B L R}=10^{3} R_{g}$.

photons: a standard accretion disk, a dusty torus in thermal equilibrium and a broad line region (BLR) modeled as a spherical shell of clouds (see Figure 1).

\section{Evolution of $\Gamma_{e q}$ along the jet}

Figure 2 represents $\Gamma_{e q}$ for different configurations of external sources. Photons from the accretion disk are emitted upward which corresponds to a positive flux in the bulk rest frame $H^{*}>0$. This leads to an inverse Compton emission backward and thus a reaction force forward, which at the end is accelerating the flow ( $\Gamma_{e q}$ increases). However, because of aberration effects, the situation is more complex for the dusty torus and the BLR. Until a certain altitude, photons from the dusty torus or from the BLR generate a negative rest frame flux, $H^{*}<0$ which produces a backward force, or Compton drag, decelerating the flow. It is only when $H *>0$ that the flow is accelerated again.

\section{References}

Boutelier, T., Henri, G., \& Petrucci, P.-O. 2008, MNRAS, 390, L73

Cheng, A. Y. S. \& O'Dell, S. L. 1981, ApJ, 251, L49

Cohen, M. H., Cannon, W., Purcell, G. H., et al. 1971, ApJ, 170, 207

Ghisellini, G., Maraschi, L., \& Treves, A. 1985, A\&A, 146, 204

Hardcastle, M. J., Kraft, R. P., Sivakoff, G. R., et al. 2007, ApJ, 670, L81

Marscher, A. P. 1980, ApJ, 235, 286

Sol, H., Pelletier, G., \& Asseo, E. 1989, MNRAS, 237, 411 